\title{
A Discrete Event Simulation of the Joint Space Cell
}

\author{
J. Mukerjee ${ }^{\text {a, }}$, Nguyen $^{\text {a }}$, R. Gani ${ }^{\text {a }}$ \\ ${ }^{\mathrm{a}}$ Defence Science and Technology Organisation, 506 Lorimer Street, Fishermans Bend, VIC 3207 \\ Email: joyanto.mukerjee@dsto.defence.gov.au
}

\begin{abstract}
The Australian Defence Force (ADF) has formed a joint space cell (JSC), to develop a set of information products to provide space situational awareness (SSA) for the Australian Defence Organisation. The JSC responds to information requests that can arrive from any agency or group within Defence. The ADF is considering different resource allocation options to enhance the capabilities offered by the JSC. To this end, we are investigating the characteristics of the relationship between the quantity of allocated resources and the rate of output through the following analytical questions:
\end{abstract}

1. What are the expected waiting times of customers requesting SSA products, given the current production process and operator allocation?

2. How many operators could be efficiently utilised within the JSC, given the existing processes for producing SSA products?

3. Is it possible to achieve significant reductions to customer waiting times through the automation of a subset of SSA production processes?

This paper reports on problem formulation, modelling and simulation approach, data acquisition and interim results. We have taken a queueing systems approach to model the operational mechanics of the JSC. We treat the operation of the JSC as a manufacturing process for information products. The JSC produces a portfolio of products and operates with a single queue and multiple servers. We have used the JSC's email traffic to characterise one of the arrival parameters of the queue. The service time that each customer experiences depends upon the product that is requested.

The results from the email analysis indicate that the exponential distribution may be used to model the daily arrival of emails into the JSC. The simulation output indicates a combination of deterministic and stochastic behaviour which is consistent with the use of the triangular distribution for generating service times.

Preliminary results indicate that DES is a reasonable approach for modelling the JSC and will provide a capability to aid decision making.

Keywords: Discrete event simulation (DES), queueing, email analysis 


\section{INTRODUCTION}

The Defence White Paper 2013 states that: "Space-based systems are a critical enabler of a modern networked military capability" (Defence (2013), p. 80). The ADF has created a Joint Space Cell (JSC) within the Joint Operations Command and tasked it to produce a set of information products that provide the ADF warfighter ${ }^{1}$ and Australian Defence Organisation ${ }^{2}$ (ADO) with space situational awareness (SSA). The JSC services the requests of ADO customers through a set of modes. The requests instigate activities that includes the production of SSA products, which are distributed to the ADO through various mechanisms (Figure 1). "SSA provides decision makers with timely and actionable information that enables the maintenance of a friendly force space advantage" (Defence (2010), p. 4-5). This makes the JSC a vital enabler in the utilisation of space based products and services by the ADF.

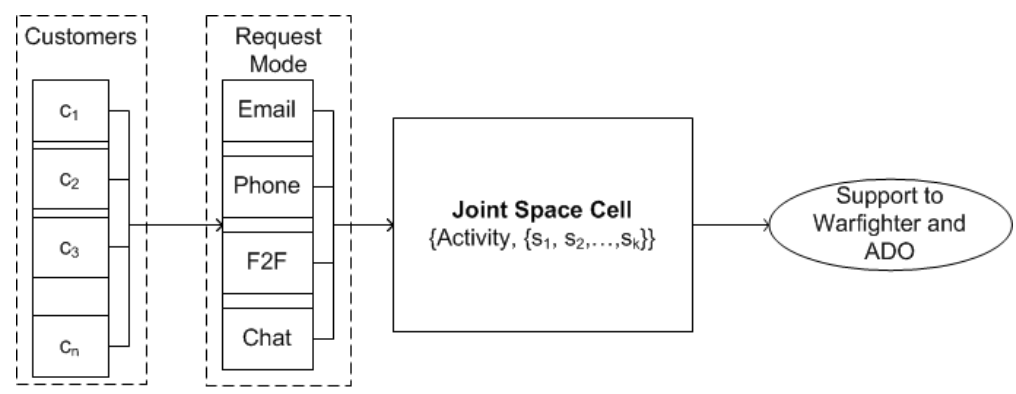

Figure 1. High level view of the JSC

The JSC is in its initial phase of operation and the Defence Science and Technology Organisation (DSTO) is conducting analysis to inform Defence about enhancing the capabilities offered by the JSC. Observation and discussion of the JSC operation with local operators has revealed two characteristics: processes used by the JSC may be treated as a business process chain for producing SSA products, allowing the JSC to be analysed through discrete event modelling (Paul et al. (1999), Curtis et al. (1992)); and, the JSC is on an evolutionary pathway towards steady state operation so any analytical approach employed needs to be resilient to organisational changes. As the JSC provides real-time support to the warfighter, experiments that are conducted in-situ would not be practical because of the disruption it may cause to normal operation. Therefore we will use a problem solving approach that is described in standard operations research text books as problem definition, data gathering, modelling, verification and validation, experimentation, analysis and documentation. The model in this case is a computer-based simulation of the JSC. Once verified and validated, the model will be subjected to experimentation that will aim to answer the following questions:

1. What are the expected waiting times of customers requesting SSA products, given the current production process and operator allocation?

2. How many operators could be efficiently utilised within the JSC, given the existing processes for producing SSA products?

3. Is it possible to achieve significant reductions to customer waiting times through the automation of a subset of SSA production processes?

\section{Method}

The analysis being undertaken is situated within the intersection of process modelling and queueing systems which suggests Business Process Simulation (BPS) as a candidate methodology (Pidd (1992)). We are aware that Defence differs from commercial enterprises in that gains in process efficiency (e.g. cost) do not always satisfy Defence requirements (e.g. safety), however the JSC clearly presents as an analogue to a service provider organisation. Given the characteristics of the JSC, its operational status and the analysis questions, the discrete event simulation (DES) method was chosen for this analytical task. BPS, rather than other static modelling techniques, allows for interaction with the model in order to analyse alternative scenarios or answer 'what if' questions (Houston (2006)). Simulation facilitates modelling with different settings of parameter values, such as resource levels, arrival rates or service times, which can help discover potential process bottlenecks (Hlupic and Robinson (1998)). A simulation model can be modified easily to follow changes in the real system and thus can be used as a decision support tool for process improvement (Hlupic and Robinson

\footnotetext{
${ }^{1}$ Member of the ADF who is engaged in the conduct of military operations.

${ }^{2}$ The whole of the Defence organisation.
} 
(1998)). Another advantage of this method is the ability to visually animate the modelled systems to facilitate communication with the JSC, both to validate the model and to explain the potential process redesign (Greasley (2003)). DES, as opposed to other simulation techniques such as systems dynamics or agent-based modelling, has been used at a more operational and tactical level to answer specific questions, e.g. to solve resource allocation problems. It is the most appropriate method to model stochastic processes and queuing systems (Brailsford and Hilton (2001)) such as this one.

The construction of a DES model of the JSC operation is underway. The current phase of model development employs an interwoven effort of data analysis and DES construction. This paper reports on the problem transformation, input-data analysis, progress on model development (including preliminary results) and possible future work.

\subsection{Problem Description}

The JSC offers a set of SSA products, $\mathrm{S}=\left\{s_{1}, s_{2}, \ldots, s_{k}\right\}$, where $k$ is a known integer (Figure 1 ). Each product is fabricated using the process chain (Figure 2) containing actors in the form of human operators that interact with computers and data providers, to complete the various roles required within process elements and produce the artifacts (SSA products) (Paul et al. (1999)). SSA products are created to satisfy a request that results from an exogenous source (e.g. ADO customer), or an endogenous requirement that must be completed to assure sustainment of capability. The set of exogenous modes $\mathrm{M}=$ \{email, phone calls, face-to-face, intranet chat message $\}$ are used by the set of customers $\mathrm{C}=\left\{c_{1}, c_{2}, \ldots, c_{n}\right\}$ to make requests, where $n$ is an unknown integer and $n \in \mathbf{Z}^{+}$. The set of arrival events is given by $\mathrm{A}=\left\{a_{1}, a_{2}, \ldots, a_{j}\right\}$ where $j$ is an unknown integer and $j \in \mathbf{Z}^{+}$. Each $a_{j}$ has unique attributes of \{arrival time and date, M, C, S, request lifetime $\}$ (Figure 1 ).

The number of human operators and available computers to generate artifacts is limited. The time taken to produce the artifacts varies stochastically and is influenced by resource availability, dependence on third party data sources and artifact complexity. The stochastic nature of arrivals and artifact production (service times) gives rise to the probability of customer queues forming at request entry points. The problem space is therefore expressed as a process model with a single queue and multiple servers (Figure 2).

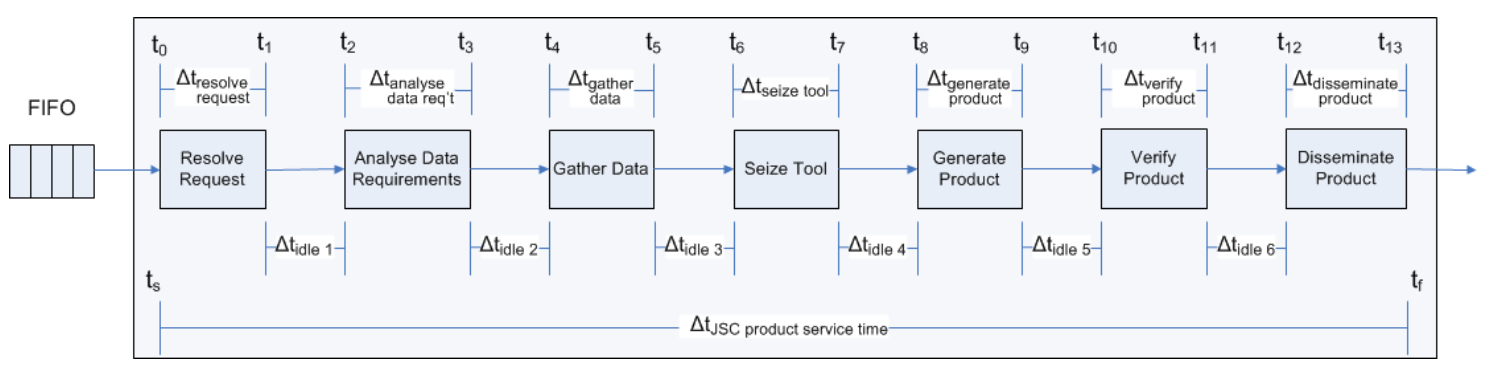

Figure 2. Generic SSA process model

The production process contains stochastic times for the completion of discrete process elements and the inter-process element idle time. Some of the factors that contribute to the stochasticity are:

- human operator/s required for completing the process element;

- process elements may have external dependencies that behave stochastically; and

- process chain occurring over scheduled or un-scheduled breaks for a given operator.

The statistical distributions that are yet to be defined for this analysis are:

- process element and idle times;

- daily operator availability; and

- unscheduled interruptions to resource availability.

The trailing edge of process completion causes the release of resources that can then be seized to process the next request within the queue. The product service time is the total time spent by a request within the production process. The arrival of requests is described by the members in set A and causes the JSC processes to be activated. The parameters being incorporated into the model are given in Table 1 
Table 1. Model parameters

\begin{tabular}{|c|c|c|}
\hline Component & Property & Quantity \\
\hline \multirow[t]{3}{*}{ Process Model } & Process Element & $\Delta \mathrm{t}_{\text {process element }}$ given by $F(t)=p(T<t)$ \\
\hline & Inter Element Gap & $\Delta \mathrm{t}_{\text {inter element idle time }}$ given by $F(t)=p(T<t)$ \\
\hline & Actor & Discrete distribution $p(X=x),\{x=0,1,2, \ldots, i\}$ \\
\hline \multirow[t]{5}{*}{ Queuing } & Arrival & Discrete distribution $p(A(t)=a),\{a=0,1,2, \ldots, j\}$ \\
\hline & Service & Process Element + Inter Element Gap \\
\hline & Servers & Discrete distribution $p(X=x),\{x=0,1,2, \ldots, k\}$ \\
\hline & Capacity & infinite \\
\hline & Discipline & FIFO \\
\hline
\end{tabular}

\subsection{Transformation into a discrete event simulation model}

The JSC operation is being modeled as a non-terminating system. It is being constructed as a data-driven model with an external parameter input file. The model will contain two sections, which are:

1. The unscheduled arrival and servicing of requests. This component specifies the number of requests that may arrive on a given day and the service times that are applied through the process model to satisfy the requests.

2. The operational conditions that are imposed upon the JSC on a given day. This influences the performance of the JSC through mandatory tasks and allocating the operators that may be available.

The approach for simulating the operation of the JSC is similar to that mentioned by Hlupic and Robinson (1998). An iterative approach is being used, which starts with the construction of a simple black box model, represented as rectangular stochastic process boxes in Figure 3 . Complexity will be increased incrementally until a model that is fit for the purpose of experimentation is obtained. The black box modeling approach is applied by representing the artifact production chains as single process elements, which use an aggregate product service time as stated in the model parameters (Table 11). When using an aggregate product service time, the time delays caused by third party dependency and resource interruption can be effectively represented through probability distributions. The current model uses operator estimates of service times for the black box processes, incorporating the service times within the model using a triangular distribution ( $\operatorname{Law}(2012))$.

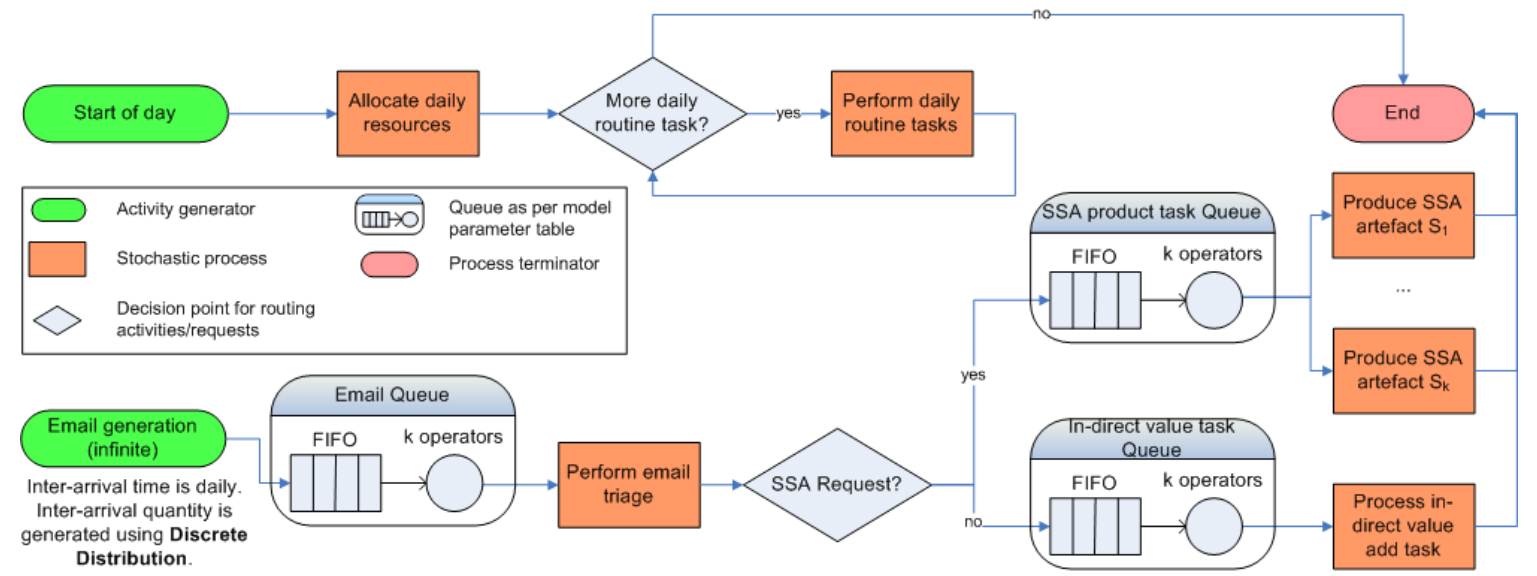

Figure 3. Logical model flowchart

\section{Data Analysis}

This section reports on the collection and preparation of data for input into the DES model. We have engaged with the JSC through observation during exercises, on-site discussion and facilitated decision conferencing. Each activity has contributed to developing an awareness of the process used by the JSC operators to satisfy ADO requests. Data is one of the primary requirements for applying the simulation methodology (Pooch and Wall Jr (1993)). Our data requirements concern the understanding of the discrete processes used, the resources that are consumed and the stimuli that cause activity to take place. Table 2 provides a summary of the current 
and planned status of data collection to satisfy the requirements for this work.

Table 2. Data collection methods

\begin{tabular}{|c|c|c|c|}
\hline Data component & Attribute & $\begin{array}{l}\text { Current data collec- } \\
\text { tion method }\end{array}$ & Options for planned data collection \\
\hline \multirow[t]{4}{*}{ Activity generation } & Email & Direct sample & Direct sample and operator estimate \\
\hline & Phone calls & None & Observation and operator estimate \\
\hline & Face-to-face & None & Observation and operator estimate \\
\hline & $\begin{array}{l}\text { Intranet chat mes- } \\
\text { sage }\end{array}$ & None & $\begin{array}{l}\text { Direct sample, observation and operator } \\
\text { estimate }\end{array}$ \\
\hline Processes & $\begin{array}{l}\Delta \mathrm{t}_{\text {process elements }} \\
\Delta \mathrm{t}_{\text {process idle times }}\end{array}$ & $\begin{array}{l}\text { Operator estimate } \\
\text { None }\end{array}$ & $\begin{array}{l}\text { Observation and operator estimate } \\
\text { Observation and operator estimate }\end{array}$ \\
\hline
\end{tabular}

The sampling of electronic mail arriving in the JSC inbox serves as a robust data sample for activity stimulus, due to the associated data attributes that are contained in every email. The analysis process for email (Figure 4) begins with executing bespoke code on the collection of emails (Extract email data) to extract the set of attributes Item type, Message type, Folder, From, To, CC, Subject, Received date, Received time, Body, Attachments $\}$ from each email. The code produces a matrix where the attribute data from each email is captured within a unique row. Each attribute is contained within a unique column. The subject and body for each email is analysed manually to identify the topic(s) and request(s). The data file is then updated to contain the assignment of topics (Assign email topics) and requests (Identify requests within emails) for each email entry. The final data file is read by a Matlab algorithm that operates on the fields $\{$ Received date, Topic, Request $\}$. The algorithm performs calculations that yield the discrete distributions for the arrival of email and the related topic (Figure 5). The input data processing captures the data within MS Excel, as it is a convenient format for the DES tool (ARENA) (Kelton et al. (2002)).

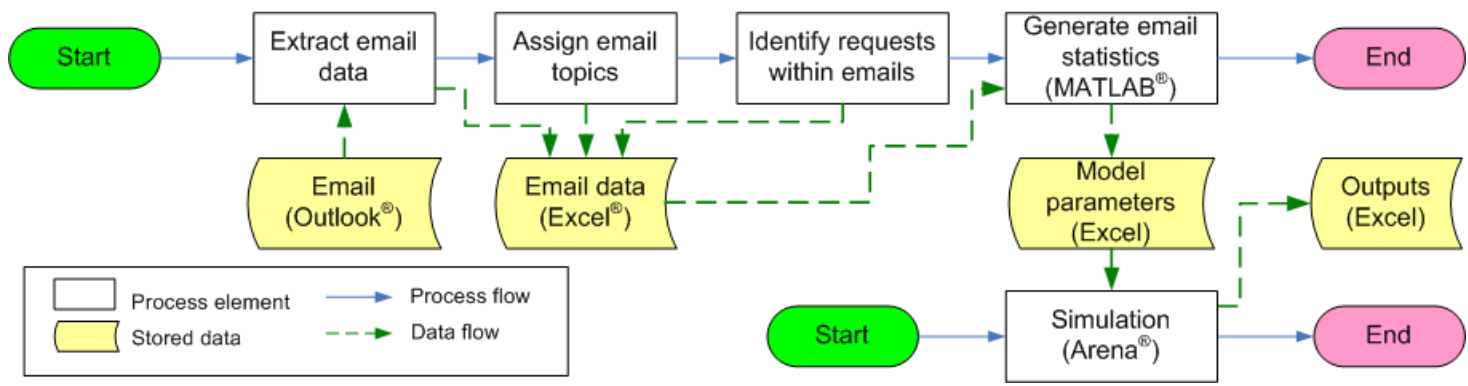

Figure 4. Data preparation and exploitation flowchart

Experimentation is performed on the simulation model and appropriate measures are recorded as output. The output is collected over a daily interval. The measures include:

- The total number of email arrivals per day.

- The number of requests by type per day.

- The time in system for each request by type in hours.

- The count of products departures by type per day.

- The amount of busy hours for each operator per day.

These measures are used to compute the rate of throughput for each request and the percentage of utilisation of each operator. The throughput is measured by computing a distribution of time in system for each request by type and the amount of SSA product by type that is completed per hour. The utilisation is computed as (busy hours / scheduled hours) $\times 100$.

\section{Preliminary Results}

Results from the analysis of emails and initial simulation runs is presented here with samples of email arrival statistics and DES output (Figure 5). The cumulative distribution function (CDF) for discrete email arrivals has been superimposed on the CDF of a fitted exponential distribution for email arrivals (Figure 5(a)). These results indicate that an exponential distribution may be used to model the arrival of emails into the JSC, underpinning a possible analytic approach to future analysis. 
The simulation output indicates a combination of deterministic and stochastic service rates, with a point where higher quantity of product departures have diminishing probability (Figure 5(b)). This result is consistent with the current model using the triangular distribution for generating service times.

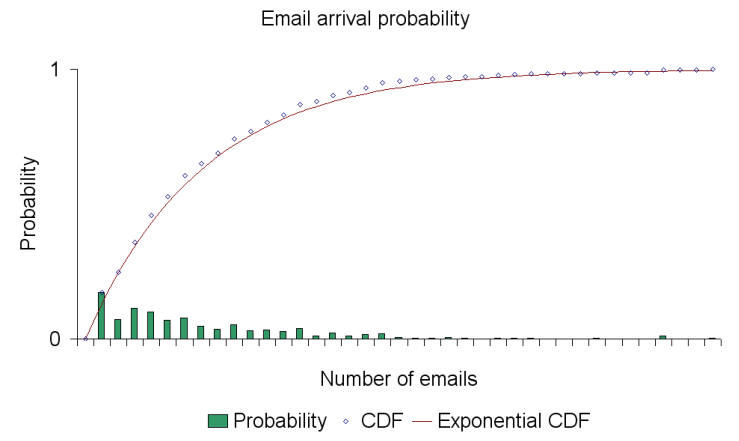

(a) Email arrival probability

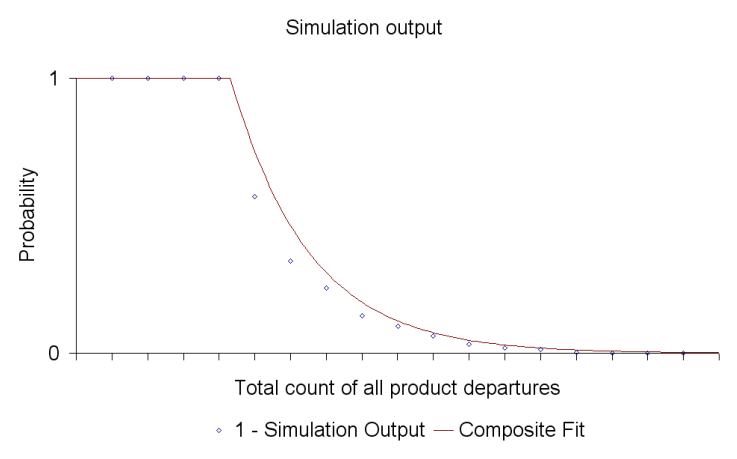

(b) Sample DES output

Figure 5. Results for current input data analysis and simulation output

\section{Discussion}

During the design phase we considered model resilience as a significant issue. We have recognised the importance of retaining situational awareness of the activities within the JSC and the process evolution during the design and construction of the model. The cell is constantly responding to changes in the ADF environment. We are making the model resilient to the changes in operational conditions of the JSC by maximising the use of model parameterisation.

Initially a coarse "daily" setting was used in which the emails arrived as a block at midnight. This skewed the results toward the JSC being able to clear their workload without the effects of resource idle time during the day. To get a more realistic effect, the arrival of emails follow one of two distributions (weekday or weekend) that depend upon the historical arrival pattern of the day in question.

The quasi deterministic nature of the DES output indicates the need for characterising the sensitivity of our measures against different service time distributions. We need to understand whether the transition between deterministic and stochastic behaviour is due to factors other than the use of triangular distributions (e.g. process mechanics). This will most probably require experimentation with higher fidelity service time distributions for the SSA production processes.

On the topic of validation we have been considering the best use of available JSC process data. Each element of JSC process data cannot be used for both defining model behaviour during construction and assessing model behaviour during validation. This creates a trade-off between increasing model accuracy (or fidelity) and model validation.

It is important that while the model is being used to support decision-making for the JSC, the assumptions and behaviours of the model are re-evaluated on a regular basis. This ongoing maintenance of the model should be recognised as a cost integral to using the model - not as an additional burden.

\section{CONCLUSION AND FUTURE DIRECTIONS}

The primary goal for the immediate future is to complete the construction and validation of the model, such that it enables experimentation and analysis to inform senior decision makers on capability enhancement for the JSC. The analysis of JSC business process mechanics may be extended to explore the consequence of the following effects:

- Switching to a priority based queueing discipline.

- Using a portfolio of tasking and technical competency of the actors used within the model.

- Using a portfolio of resource availability and request arrival rates that depend upon on a set of operational conditions.

Our engagement with the JSC, analysis of emails, process mechanics and DES output data indicate that the current simulation approach can accommodate our requirements for modelling the JSC. The model animation 
is used as a tool for communicating our work to the JSC during status update meetings. The ongoing evolution of the modelling approach will yield a flexible simulation model that will be used to aid Defence decision making. The analysis of email data could be used to explore the following:

- Using email and other data to indicate the change of phase in the operation of the JSC and connected entities, such as transient to steady state.

- The possibility of conducting network analysis using source to sink attributes with topic and request, to gain a broader understanding of space operations.

Consideration is being given to automation of email analysis that can approximate the concepts proposed by Bengtsson et al. (2009). A fully automated data treatment could result in a simulation based forecast model that may be used for real-time decision support (Kádár et al.(2004)).

\section{ACKNOWLEDGEMENT}

The authors would like to thank the JSC, Jason Ip, Tarsem Singh, Stephen Bocquet, Simon Ng and Ross Ashman for their generous support.

\section{REFERENCES}

Bengtsson, N., G. Shao, B. Johansson, Y. T. Lee, S. Leong, A. Skoogh, and C. Mclean (2009). Input data management methodology for discrete event simulation. In Proceedings of the Winter Simulation Conference, pp. 1335-1344. IEEE.

Brailsford, S. and N. Hilton (2001). A comparison of discrete event simulation and system dynamics for modelling health care systems. In J. Riley (Ed.), Planning for the Future: Health Service Quality and Emergency Accessibility, Volume Glasgow. Glasgow Caledonian University.

Curtis, B., M. I. Kellner, and J. Over (1992). Process modeling. Communications of the ACM 35(9), 75-90.

Defence (2010). Operations Series ADDP 3.18 Operational Employment of Space. Department of Defence, Australia.

Defence (2013). Defence White Paper. Department of Defence, Australia.

Greasley, A. (2003). Using business-process simulation within a business-process reengineering approach. Business Process Management Journal 9(4), 408-420.

Hlupic, V. and S. Robinson (1998). Business process modelling and analysis using discrete-event simulation. In Proceedings of the Winter Simulation Conference, pp. 1363-1370. IEEE Computer Society Press.

Houston, D. (2006). An experience in facilitating process improvement with an integration problem reporting process simulation. Software Process: Improvement and Practice 11(4), 361-371.

Kádár, B., A. Pfeiffer, and L. Monostori (2004). Discrete event simulation for supporting production planning and scheduling decisions in digital factories. In Proceedings of the 37th CIRP international seminar on manufacturing systems, pp. 444-448.

Kelton, W. D., R. P. Sadowski, and D. A. Sadowski (2002). Simulation with ARENA, Volume 2. McGraw-Hill New York.

Law, A. M. (2012). A tutorial on how to select simulation input probability distributions. In Proceedings of the Winter Simulation Conference, pp. 1-15. IEEE.

Paul, R. J., G. M. Giaglis, and V. Hlupic (1999). Simulation of business processes. American Behavioral Scientist 42(10), 1551-1576.

Pidd, M. (1992). Computer simulation in management science. John Wiley \& Sons, Inc.

Pooch, U. W. and J. A. Wall Jr (1993). Discrete event simulation: a practical approach, Volume 4. CRC Press. 